\title{
A Burden Profile Meter in Blast Furnace Using a New Type of Microwave Radar*
}

\section{Introduction}

It is important in blast furnace operations to control the distribution pattern of charged raw materials to a desired pattern in order to stabilize the operation of the blast furnace and improve its efficiency. Several kinds of burden level meters using microwave have been reported. But the configurations of these radars are complex and large in size due to a wide frequency band $(\Delta F>500 \mathrm{MHz})$.

To improve these problems, a narrow band frequency-modulated (FM) radar based on a new phase modulation method has been developed and applied to a burden profile meter.

\section{Configuration of Equipment}

The overall structure of the prototype profile meter is shown in Fig. 1. This equipment consists of a radar unit, a signal processing unit and a moving lance designed to drive the radar unit from the wall to the center of the blast furnace.

The microwave circuit is arranged in a water-cooled jacket so as to protect each device from hightemperature gas in the blast furnace. Furthermore, dust purge means is adapted to feed inert gas to the antenna in order to avoid possible deposition of dust on the antenna. In order to minimize the size and weight of the radar unit, the microwave circuit is simply composed and operated under conditions of high frequency (24.1 $\mathrm{GHz}$ ) and narrow band width $(\Delta F=50 \sim 100 \mathrm{MHz})$.

\section{Principle of Measurement}

The principle of measurement is based on the FM method. In this method a frequency-modulated microwave is transmitted from the radar toward the burden, and a reflected wave is received. Both the transmitted and received wave are mixed in a diode mixer and a so-called beat signal is obtained and led to the signal processing unit.

In the signal processing unit, modulation is applied to the phase fraction of each beat wave for decreasing the step error in FM radar. In this method, two sine waves of angular frequency $\omega_{1}$ and $\omega_{2}$ are mixed to the beat signal, and the phase of the beat signal is modulated to a degree of $2 \pi$ in a period of $2 \pi /\left(\omega_{1}-\omega_{2}\right)$. During one cycle of the modulation, the zerocrossing counts of the beat signal are averaged and so the step error is reduced $(450 \rightarrow$ $18 \mathrm{~mm}$ ).

\section{Experimental Result}

For the purpose of estimating the measurement error for burden raw materials, levels of coke were measured in the laboratory. The standard deviation of the error was $50 \mathrm{~mm}$, and so it was found to be sufficiently accurate for a burden profile meter.

One of the experimental results carried out at a blast furnace is shown in Fig. 2. The burden profile measured by this equipment was verified to be similar to the results obtained by the mechanical method. This equipment is now operating successfully in a blast furnace at Kakogawa Works of Kobe Steel, Ltd.

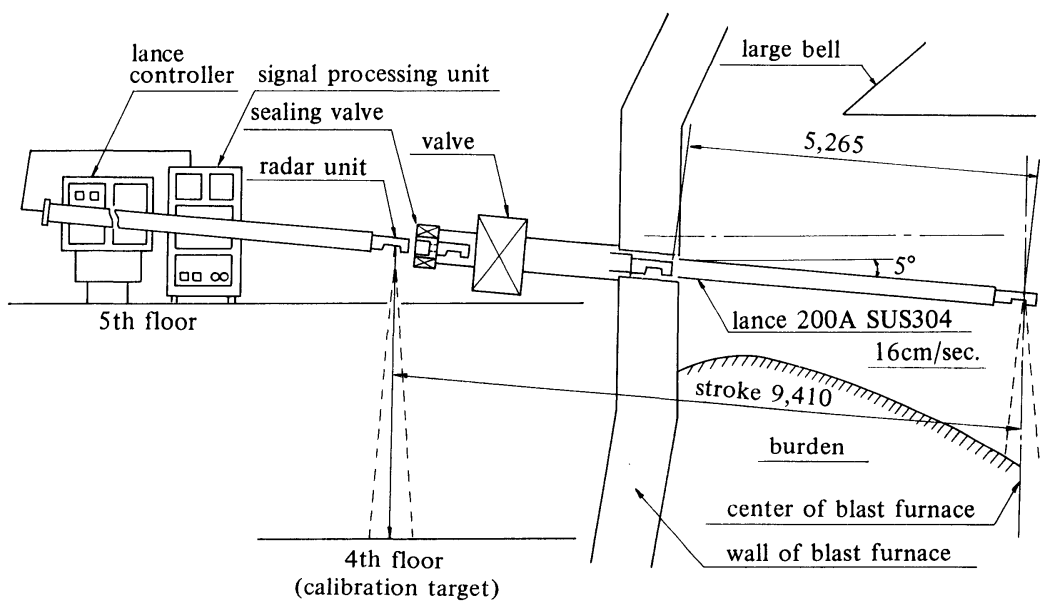

Fig. 1. Microwave level meter for blast furnace.

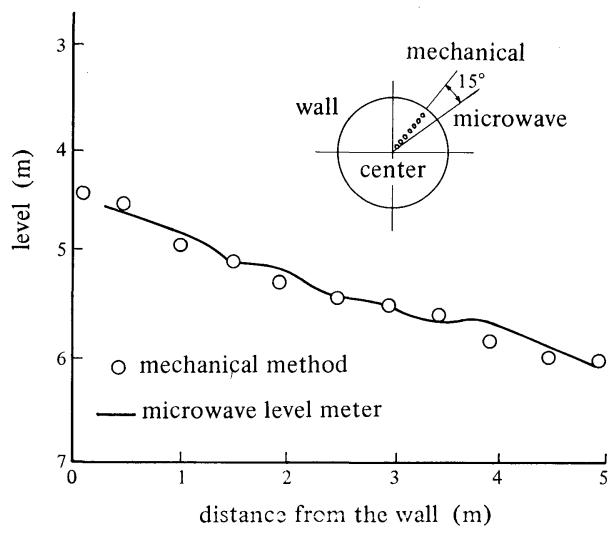

Fig. 2. Compared result with mechanical method during blowing down.

\footnotetext{
* For further information, write to Asada Research Laboratories, Kobe Steel, Ltd., 53-3 Gomo Aza Maruyama, Nada-ku, Kobe 657.
} (C) 1986 ISIJ 